\title{
LETTER
}

\section{Effect of erythropoietin therapy on clinical outcome in patients after acute ischemic stroke: a debatable issue}

\author{
Chun-Man Yuen', Cheuk-Kwan Sun ${ }^{2,3}$, Steve Leu ${ }^{4,5}$ and Hon-Kan Yip*4,5 \\ See related research by Yip et al., http://ccforum.com/content/15/1/R40, and related commentary by Minnerup et al., \\ http://ccforum.com/content/15/2/129
}

Our recent clinical trial has shown that erythropoietin (EPO) therapy significantly increased circulating endothelial progenitor cell (EPC) levels and was strongly associated with favorable 90-day clinical outcomes after ischemic stroke (IS) [1]. Contrary to the findings of our study [1] and of others [2,3], the randomized phase II/III German Multicenter EPO Stroke Trial [4], which is currently the largest clinical study on EPO treatment in patients with IS, not only failed to show any additional benefit but also demonstrated increased mortality after combined therapy with EPO and tissue plasminogen activator (tPA) [4]. Interestingly, subgroup analysis of the study revealed that EPO therapy improved 90-day clinical outcome in patients without additional tPA therapy [4]. In this way, the findings from the subgroup analysis of that study [4] corroborate those of our clinical trial [1].

We thank Minnerup and colleagues [5] for their comments in the previous issue of Critical Care. These authors suggested that, for evaluating 90-day neurological outcome, the modified Rankin Scale or the Barthel Index could be superior to the National Institutes of Health Stroke Scale (NIHSS) that was used in our study [5]. They also mentioned that, when the components of the composite endpoint in our study protocol are considered, the significantly higher number of patients with an NIHSS score of at least 8 after placebo treatment is also likely to be caused by the high rate of recurrent strokes and does not necessarily reflect improved neurological function in the EPO group [5]. However, NIHSS is widely accepted as one of the most efficacious tools for evaluating neurological outcome after acute IS. Additionally,

*Correspondence: han.gung@msa.hinet.net

${ }^{4}$ Division of Cardiology, Department of Internal Medicine, Chang Gung Memorial Hospital - Kaohsiung Medical Center, Chang Gung University College of Medicine, 123 Ta-Pei Road, Niaosong District, Kaohsiung City, 833, Taiwan

Full list of author information is available at the end of the article the trial in our study was prospective, randomized, and placebo-controlled - this is the best design to minimize the selection bias between the study group and the control group. Accordingly, we suggest that the significantly lower number of patients with an NIHSS score of at least 8 in the EPO group in comparison with the placebo group could reflect simply the therapeutic benefit of EPO therapy in improving the 90-day neurological outcome rather than a mere speculation of a higher rate of recurrent strokes in the placebo group.

The optimal dosage of EPO and duration of treatment for patients after IS remain uncertain. This may account for some inconsistency in improvement of neurological outcome after IS in clinical trials [1-4]. Accordingly, we disagree with Minnerup and colleagues [5] that the potential side effects and the failed efficacy in large clinical trials will presumably prevent the use of EPO as a therapy to increase EPCs after stroke.'

Minnerup and colleagues also underscored that the study was first assigned to a trial register (ISRCTN 96340690) in January 2011, which was 10 months after the inclusion of the last patients in March 2010 [5]. We would like to underscore that, in addition to receiving institutional review board approval, our study [1] was reviewed and approved by the National Science Council of Taiwan before implementation. Actually, our clinical trial started long before we applied for an assignment to a trial registry. We believe that well-controlled studies remain the primary bases of scientific progress. Further evidence is required for solid conclusions to be made.

\section{Abbreviations}

EPC, endothelial progenitor cell; EPO, erythropoietin; NIHSS, National Institutes of Health Stroke Scale; IS, ischemic stroke; tPA, tissue plasminogen activator.

\section{Competing interests}

The authors declare that they have no competing interests.

\section{Authors' contributions}

C-MY and SL wrote the manuscript. H-KY and C-KS revised the manuscript. All authors read and approved the final manuscript. 


\section{Author details}

'Division of Trauma, Department of Surgery, Chang Gung Memorial Hospital Kaohsiung Medical Center, Chang Gung University College of Medicine, 123 Ta-Pei Road, Niaosong District, Kaohsiung City, 833, Taiwan. ${ }^{2}$ Division of General Surgery, Department of Surgery, Chang Gung Memorial Hospital Kaohsiung Medical Center, Chang Gung University College of Medicine, 123 Ta-Pei Road, Niaosong District, Kaohsiung City, 833, Taiwan. ${ }^{3}$ Department of Emergency Medicine, E-Da Hospital, I-Shou University, Kaohsiung City, 824, Taiwan ${ }^{4}$ Division of Cardiology, Department of Internal Medicine, Chang Gung Memorial Hospital - Kaohsiung Medical Center, Chang Gung University College of Medicine, 123 Ta-Pei Road, Niaosong District, Kaohsiung City, 833, Taiwan. ${ }^{5}$ Center for Translational Research in Biomedical Sciences, Chang Gung Memorial Hospital - Kaohsiung Medical Center, Chang Gung University College of Medicine, 123 Ta-Pei Road, Niaosong District, Kaohsiung City, 833, Taiwan.

Published: 17 May 2011

\section{References}

1. Yip HK, Tsai TH, Lin HS, Chen SF, Sun CK, Leu S, Yuen CM, Tan TY, Lan MY, Liou CW, Lu CH, Chang WN: Effect of erythropoietin on level of circulating endothelial progenitor cells and outcome in patients after acute ischemic stroke. Crit Care 2011, 15:R40.

2. Tseng MY, Hutchinson PJ, Richards HK, Czosnyka M, Pickard JD, Erber WN, Brown S, Kirkpatrick PJ: Acute systemic erythropoietin therapy to reduce delayed ischemic deficits following aneurysmal subarachnoid hemorrhage: a Phase II randomized, double-blind, placebo-controlled trial. Clinical article. J Neurosurg 2009, 111:171-180.

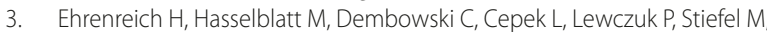
Rustenbeck HH, Breiter N, Jacob S, Knerlich F, Bohn M, Poser W, Rüther E, Kochen M, Gefeller O, Gleiter C, Wessel TC, De Ryck M, Itri L, Prange H, Cerami A, Brines M, Sirén AL: Erythropoietin therapy for acute stroke is both safe and beneficial. Mol Med 2002, 8:495-505

4. Ehrenreich $\mathrm{H}$, Weissenborn $\mathrm{K}$, Prange $\mathrm{H}$, Schneider D, Weimar C, Wartenberg K, Schellinger PD, Bohn M, Becker H, Wegrzyn M, Jähnig P, Herrmann M, Knauth M, Bähr M, Heide W, Wagner A, Schwab S, Reichmann H

Schwendemann G, Dengler R, Kastrup A, Bartels C; EPO Stroke Trial Group: Recombinant human erythropoietin in the treatment of acute ischemic stroke. Stroke 2009, 40:e647-656

5. Minnerup J, Wersching H, Schäbitz W: Erythropoietin for stroke treatment: dead or alive? Crit Care 2011, 15:129.

doi:10.1186/cc10145

Cite this article as: Yuen C-M, et al:: Effect of erythropoietin therapy on clinical outcome in patients after acute ischemic stroke: a debatable issue. Critical Care 2011, 15:425. 